\title{
PLEK2 promotes osteosarcoma tumorigenesis and metastasis by activating the PI3K/AKT signaling pathway
}

\author{
YANG LIU $^{1 *}$, SITING YANG ${ }^{2 *}$, FENG WANG $^{3 *}$, ZHENG ZHOU $^{4 *}$, \\ WENJING XU ${ }^{5}$, JINGJING XIE ${ }^{5}$, LINHUI QIAO ${ }^{1}$ and YANGLIN GU ${ }^{1}$
}

\begin{abstract}
${ }^{1}$ Department of Orthopedics, The Affiliated Wuxi No. 2 People's Hospital of Nanjing Medical University, Wuxi, Jiangsu 214002; ${ }^{2}$ Department of Anesthesiology and Nursing, The First Affiliated Hospital of Nanjing Medical University, Nanjing, Jiangsu 210029; ${ }^{3}$ Department of Analysis Center, Nanjing Medical University, Nanjing, Jiangsu 211166; ${ }^{4}$ Department of Orthopedics, The First Affiliated Hospital of Nanjing Medical University, Nanjing, Jiangsu 210029; ${ }^{5}$ Department of Ultrasound, Wuxi Xishan People's Hospital, Wuxi, Jiangsu 214000, P.R. China
\end{abstract}

Received February 20,2021; Accepted April 28, 2021

DOI: 10.3892/ol.2021.12795

\begin{abstract}
Increasing evidence suggest that pleckstrin-2 (PLEK2) acts as an oncogene in several malignancies. The present study aimed to investigate the effects of PLEK2 on osteosarcoma (OS) tumorigenesis and metastasis. PLEK2 expression in OS was analyzed via bioinformatics, reverse transcription-quantitative PCR, western blot and immunohistochemistry analyses. The Cell Counting Kit-8 (CCK-8), colony formation and EdU assays were performed to assess the role of PLEK2 in OS cell proliferation. The pro-metastatic effects of PLEK2 were assessed via the Transwell and wound healing assays. In addition, the PLEK2 downstream pathway was analyzed via bioinformatics analysis and verified via western blot analysis. The results demonstrated that PLEK2 expression was upregulated in both OS cell lines and specimens. The results of the CCK-8, colony formation and EdU assays demonstrated that PLEK2 promoted OS cell proliferation in vitro. The in vivo experiments further demonstrated that PLEK2 knockdown significantly suppressed OS growth. In addition, the Transwell and wound healing assays indicated that PLEK2 promoted OS invasiveness in vitro, which was induced by the activation of the epithelial-to-mesenchymal transition process. Bioinformatics analysis revealed that PLEK2 can activate the phosphoinositide 3-kinase (PI3K)/ protein kinase $\mathrm{B}(\mathrm{AKT}) /$ mechanistic target of rapamycin (mTOR) pathway, which was verified via western blot analysis. Taken together, the results of the present study suggest that
\end{abstract}

Correspondence to: Dr Yanglin Gu, Department of Orthopedics, The Affiliated Wuxi No. 2 People's Hospital of Nanjing Medical University, 68 Zhong Shan Road, Wuxi, Jiangsu 214002, P.R. China E-mail: wxeygyl@163.com

${ }^{*}$ Contributed equally

Key words: Pleckstrin-2, osteosarcoma, proliferation, metastasis, PI3K/AKT signaling pathway
PLEK2 may play a tumor-promoting role in OS via the PI3K/AKT signaling pathway.

\section{Introduction}

Osteosarcoma (OS) is a primary malignant bone tumor typically diagnosed in children and young adults, and patients may suffer from severe pain or other symptoms associated with pathological fractures (1). Distant metastases have been reported in $\sim 15-20 \%$ of patients diagnosed with OS (2). Current therapies for OS include surgical resection and combined chemotherapy, which cure $\sim 70 \%$ of cases (3). However, the overall 5-year survival rate for patients with OS is estimated at $20 \%$, and the survival rates for patients with distant metastatic or relapsed OS have not improved for the past 30 years $(4,5)$.

Tumor metastasis is associated with the tumor microenvironment, which plays a key role in the progression of malignant tumors (6). The epithelial-to-mesenchymal transition (EMT) process is a crucial mechanism in the progression of tumor metastasis and is characterized by the loss of cell-cell adhesions and the acquisition of cell-matrix interactions (7). EMT is involved in the formation of several tissues and organs during embryonic development, in addition to tumor invasion and metastases (8).

Pleckstrin-2 (PLEK2) is a 353 amino acid protein that is expressed in a variety of tissues (9). PLEK2 expression has been implicated in breast cancer cell dissemination and is negatively associated with clinical outcomes (10). Notably, PLEK2 has been suggested as a potential gene marker for distinguishing the CD45 subgroup between patients with melanoma and healthy individuals (11). A previous study demonstrated that PLEK2 expression in blood serves as an early biomarker for melanoma diagnosis (11), and another study provided compelling evidence that PLEK2 can promote gallbladder carcinoma cell migration, invasion and liver metastasis by regulating the EMT process (12). Although the role of PLEK2 in tumor development is gradually being recognized, the contributions of PLEK2 to OS progression have not yet been studied.

The present study aimed to investigate the role of PLEK2 in OS and determine its underlying molecular mechanisms. 
The results may provide an avenue for prospective studies and provide a potential therapeutic target for OS.

\section{Materials and methods}

Clinical tissues specimens. The age distribution of patients ranged from 14-22 years, and there were seven patients (all men) $>18$ years and thirteen patients (all men) $<18$ years. A total of 20 pairs of OS tissues and adjacent normal tissues ( $2 \mathrm{~cm}$ away from tumor) were collected during surgical resection from the Department of Orthopedics, The Affiliated Wuxi No. 2 People's Hospital of Nanjing Medical University (Wuxi, China) and the Department of Orthopedics, The First Affiliated Hospital of Nanjing Medical University (Nanjing, China). All tissues were stored at $-80^{\circ} \mathrm{C}$ until protein extraction or paraffin embedding. The present study was approved by the Ethics Committee of the Affiliated Wuxi No. 2 People's Hospital of Nanjing Medical University (Wuxi, China; approval no. 2019-01-0202-05) and written informed consent was provided by patients or their legal guardians prior to the study start.

Reagents and antibodies. The cell culture reagents, including culture medium, fetal bovine serum (FBS) and penicillin-streptomycin were purchased from Gibco; Thermo Fisher Scientific, Inc. The Cell Counting Kit-8 (CCK-8) and EdU Proliferation kits were purchased from Dojindo Molecular Technologies, Inc., and Guangzhou RiboBio Co., Ltd., respectively. The agonist (insulin-like growth factor 1) and inhibitor (LY 294002) of PI3K/AKT pathway were purchased from MedChemExpress, Inc. The primary antibodies used were against PLEK2 (ProteinTech Group, Inc.), N-cadherin (Cell Signaling Technology, Inc.), matrix metalloproteinase (MMP)2 (Cell Signaling Technology, Inc.), vimentin (Cell Signaling Technology, Inc.), PI3K (Cell Signaling Technology, Inc.), phosphorylated (p)-PI3K (Cell Signaling Technology, Inc.), AKT (Cell Signaling Technology, Inc.), p-AKT (Cell Signaling Technology, Inc.) and GAPDH (Jackson ImmunoResearch Labortaories, Inc.).

Microarray data analysis. A total of three Gene Expression Omnibus (GEO) datasets, including GSE12865 (13), GSE14359 (14) and GSE33382 (15), were downloaded from the GEO database (https://www.ncbi.nlm.nih.gov/geo), which compare the mRNA levels of OS tissues with those of normal tissues. After importing the profiles into $\mathrm{R}$ studio (version 3.6.0; RStudio, Inc.), the data were normalized and probes were nominated. Differentially expressed genes (DEGs) were identified using R package (Limma; version 3.30.11; http:// www.bioconductor.org/). Genes with llog fold changel $\geq 1$ were consdiered DEGs. To investigate the biological processes that PLEK2 participates in, the correlation coefficients between each gene and PLEK2 were calculated to perform gene set enrichment analysis (GSEA).

Cell culture. The OS cell lines, U-2 OS, HOS, Saos-2 and 143B, and human osteoblast hFOB 1.19 cells were purchased from the Cell Bank of Type Culture Collection of the Chinese Academy of Sciences. OS cells were maintained in Dulbecco's modified Eagle's medium (DMEM) supplemented with 10\% FBS and
$1 \%$ penicillin-streptomycin, at $37^{\circ} \mathrm{C}$ with $5 \% \mathrm{CO}_{2}$. The hFOB 1.19 osteoblast cells were maintained in DMEM/F-12 medium containing $0.3 \mathrm{mg} / \mathrm{ml}$ geneticin (Gibco, Thermo Fisher Scientific, Inc.), $10 \%$ FBS and $1 \%$ penicillin-streptomycin, at $33.5^{\circ} \mathrm{C}$ with $5 \% \mathrm{CO}_{2}$.

Cell transfection and lentivirus construction. To determine the biofunctions of PLEK2 in OS cells, PLEK2 overexpressing U-2 OS cells and PLEK2 knockdown 143B cells were constructed and verified for PLEK2 expression. Lentiviral vectors carrying human PLEK 2 cDNA and short hairpin RNA (shRNA) targeting PLEK2 (sh-PLEK2, 5'-CCTGGA GAATGGGAGTGTTAA-3') were constructed by Shanghai GenePharma Co., Ltd. When the 2nd cells reached $40 \%$ confluence, they were infected with vector (GenePharma Co., Ltd.), PLEK2 (GenePharma Co., Ltd.), shRNA encoding a non-specific control sequence (sh-NC, 5'-TTCTCCGAA CGTGTCACGT-3', MOI=50), or sh-PLEK2 (MOI=50), using Lipofectamine $^{\circledR} 3000$ (Invitrogen; Thermo Fisher Scientific, Inc.) overnight at $37^{\circ} \mathrm{C}$. Stably transfected cell lines were obtained by treatment with $5 \mu \mathrm{g} / \mathrm{ml}$ puromycin (Sigma-Aldrich; Merck KGaA) for 1 week before experimentation. PLEK2 mRNA and protein expression levels in stably transfected cells were validated via reverse transcription-quantitative (RT-q) PCR and western blot analyses, respectively.

CCK- 8 assay. The CCK- 8 assay was performed to assess OS cell proliferation. Stably transfected cells were seeded into 96-well plates at a density of 1,000 cells/well and allowed to grow for different time periods (every $12 \mathrm{~h}$ until $72 \mathrm{~h}$ ). At the specified time points, $10 \mu \mathrm{lCCK}-8$ reagent was added to each well. Following incubation for $2 \mathrm{~h}$, cell proliferation was detected at a wavenlength of at $450 \mathrm{~nm}$, using a spectrophotometer (Thermo Fisher Scientific, Inc.).

EdU assay. To further investigate the effects of PLEK2 on OS cell proliferation, the EdU assay was performed. Cells were seeded into 96-well plates at a density of 4,000 cells/well and allowed to grow for $24 \mathrm{~h}$. Subsequently, $50 \mu \mathrm{M}$ EdU solution was added to each well and incubated for $2 \mathrm{~h}$. Following fixation with $4 \%$ polyformaldehyde, the cells were permeated with $0.5 \%$ Triton X-100 and stained with 1x Apollo reagent for 30 min. Nuclei were stained with 1x Hoechst 33342 and cells were observed under a fluorescence microscope (magnification, x200; Carl Zeiss AG).

Colony formation assay. Stably transfected OS cells were seeded into 6-well plates at a density of 200 cells/well and allowed to grow for 2 weeks. Following incubation, cell colonies were fixed with $4 \%$ polyformaldehyde and visualized by staining with $0.1 \%$ crystal violet. The number of cell colonies was counted manually.

Transwell assay. To assess the pro-metastatic effects of PLEK2 in OS cells, the Transwell assay was performed using an insert with an $8-\mu \mathrm{m}$ pore (Corning, Inc.). A total of 10,000 cells were seeded into the upper chambers in $200 \mu \mathrm{l}$ DMEM with no FBS, and $600 \mu 1$ DMEM supplemented with $10 \%$ FBS was plated in the lower chambers. Following incubation for $24 \mathrm{~h}$, cells were fixed with $4 \%$ polyformaldehyde and stained with $0.1 \%$ crystal 
violet for $10 \mathrm{~min}$. Cells that migrated into the lower chambers were observed under a light microscope (magnification, x200), and the number of cells/field was calculated using ImageJ software (version 2.1.4.7; National Institutes of Health). For the invasion assay, Matrigel was used to pretreat the Transwell inserts at $37^{\circ} \mathrm{C}$ for $1 \mathrm{~h}$ until gel formation occurred, and the migration assay was performed using Matrigel-coated inserts.

Wound healing assay. Stably transfected cells were seeded into 6-well plates until they reached $100 \%$ confluence. A P200 pipette tip was used to scratch the cell monolayers. Cells were subsequently cultured in DMEM medium without serum to exclude the interference of cell proliferation in migration distance. At the indicated time points $(0$ and $24 \mathrm{~h}$ after scratching), the cell layers were observed under a a fluorescence microscope (magnification, $\mathrm{x} 40$ ), and the migration distance was measured to assess cell motility.

$R T$ - $q P C R$. Total RNA was extracted from cells using TRIzol ${ }^{\circledR}$ reagent (Takara, Japan), following the manufacturer's instructions. The extracted mRNA was subsequently reverse transcribed at $37^{\circ} \mathrm{C}$ for $15 \mathrm{~min}$ and $85^{\circ} \mathrm{C}$ for $5 \mathrm{sec}$ using the PrimeScript RT Reagent kit (Takara Bio, Inc.). qPCR was subsequently performed (predenaturation at $95^{\circ} \mathrm{C}$ for $10 \mathrm{~min}$, denaturation at $95^{\circ} \mathrm{C}$ for $15 \mathrm{sec}$, renaturation at $60^{\circ} \mathrm{C}$ for $60 \mathrm{sec}$ and repeat for 40 cycles) using a SYBR Green PCR master mix (Nanjing KeyGen Biotech Co., Ltd.). The following primer sequences were used for qPCR: PLEK2 forward, 5'-GTGCTCAAGGAGGGCTTC-3' and reverse, 5'-GCTTGT AGTACACCAGCGTGTT-3'; and GAPDH forward, 5'-AAT GGGCAGCCGTTAGGAAA-3' and reverse, 5'-GCGCCC AATACGACCAAATC-3'. PLEK2 expression was normalized to GAPDH and relative expression was calculated using the $2^{-\Delta \Delta \mathrm{Cq}}$ method (16).

Western blotting. Total protein of OS cells or tissues was extracted using RIPA lysis buffer (Beyotime Institute of Biotechnology) containing phenylmethylsulfonyl fluoride and phosphatase inhibitor, and protein concentration was determined using the BCA Protein Assay kit (Beyotime Institute of Biotechnology). Following denaturation at $95^{\circ} \mathrm{C}$, $30 \mu \mathrm{g}$ proteins were separated via $10 \%$ SDS-PAGE, electrically transferred onto PVDF membranes and blocked with 5\% skimmed milk at room temperature for $2 \mathrm{~h}$. The membranes were incubated with primary antibodies overnight at $4^{\circ} \mathrm{C}$, followed by incubation with HRP-conjugated secondary antibodies (Jackson ImmunoResearch Labortaories, Inc., 1:5,000, cat. no. 111-035-003) for $2 \mathrm{~h}$ at room temperature. TBST (0.1\% Tween-20) was used to wash the membranes between each incubation, and the proteins were visualized using an ECL detection kit (Tanon Science and Technology Co., Ltd.). The expression levels of all proteins were normalized to that of GAPDH and ImageJ software (version 2.1.4.7; National Institutes of Health) was used for densitometry.

Immunohistochemistry. For immunohistochemistry analysis, all specimens were embedded in paraffin and cut into $5-\mu \mathrm{m}$-thick sections. Following antigen retrieval with citrate sodium and blocking in 5\% bovine serum albumin at room temperature for $2 \mathrm{~h}$, the sections were incubated with primary antibody for PLEK2 overnight at $4^{\circ} \mathrm{C}$. The sections were incubated with the corresponding HRP-conjugated secondary antibody (Jackson ImmunoResearch Labortaories, Inc., 1:200, cat. no. 111-035-003) for $2 \mathrm{~h}$ at room temperature and visualized using 3,3'-diaminobenzidine (Beijing Solarbio Science \& Technology Co., Ltd.) for $3 \mathrm{~min}$. The sections were observed under a light microscope (magnification, x200).

Animal experiments. All in vivo animal experiments were approved by the Institutional Animal Care and Use Committee of the Affiliated Wuxi No. 2 People's Hospital of Nanjing Medical University. Mice were housed under a controlled temperature $\left(23 \pm 0.5^{\circ} \mathrm{C}\right)$, humidity $(50 \pm 10 \%)$ and a $12: 12 \mathrm{~h}$ light:dark cycle, food, drinking water and litter were changed every 2 days. A total of 16 6-week-old male nude mice (18-20 g) were randomly divided into two groups (sh-NC and sh-PLEK2, $\mathrm{n}=8$ ) and injected with stably transfected OS cells to perform subcutaneous tumorigenesis analysis. Tumor volumes were examined and calculated (Volume=length $\mathrm{x}$ width ${ }^{2} \times 0.5$ ) every 4 days after injection until mice were euthanized at day 28 post injection. All mice were enthanized by intraperitoneal injection of pentobarbital sodium $(100 \mathrm{mg} / \mathrm{kg}) 4$ weeks after injection (17). The death of mice was verified 10 min after injection by loss of movement, breath, heartbeat, corneal reflex and muscular tension. Tumor weight was measured after the tumor node was removed, and Ki-67 expression was detected via immunohistochemistry analysis.

Statistical analysis. Statistical analysis was performed using GraphPad Prism 8.0 software (GraphPad Software, Inc.). All experiments were performed in triplicate and data are presented as the mean \pm standard deviation. Unpaired Student's t-test was used to compare differences between two groups, and paired Student's t-test was used to compare differences between paired OS tissues and normal tissues. Two-way ANOVA followed by Sidak's multiple comparisons test were used to compare differences between multiple groups. $\mathrm{P}<0.05$ was considered to indicate a statistically significant difference.

\section{Results}

PLEK2 is significantly upregulated in OS cell lines and tissues. After analyzing three OS datasets using R language, the results demonstrated that PLEK2 expression was notably higher in OS tissues compared with adjacent normal tissues in all three datasets (Fig. 1A), with log fold change $>1$ (Fig. 1B). RT-qPCR and western blot analyses were performed to detect PLEK2 expression in OS cell lines and tissues. As presented in Fig. 1C ( $\mathrm{P}=0.0272$ for $\mathrm{U}-2 \mathrm{OS}, \mathrm{P}=0.0023$ for $\mathrm{HOS}, \mathrm{P}=0.0001$ for Saos-2 and 143B compared to hFOB 1.19), PLEK2 mRNA expression was significantly upregulated in OS cell lines compared with osteoblast cells, and 143B cells exhibited the highest PLEK2 expression, whereas U-2 OS cells exhibited the lowest expression. Western blot analysis further demonstrated that PLEK2 protein expression was significantly higher in the OS cell lines and tissues (Fig. 1D). In addition, immunohistochemistry analysis demonstrated that PLEK2 was highly expressed in OS tissues (Fig. 1E). Taken together, these results suggest that PLEK2 expression is upregulated in OS. 
A

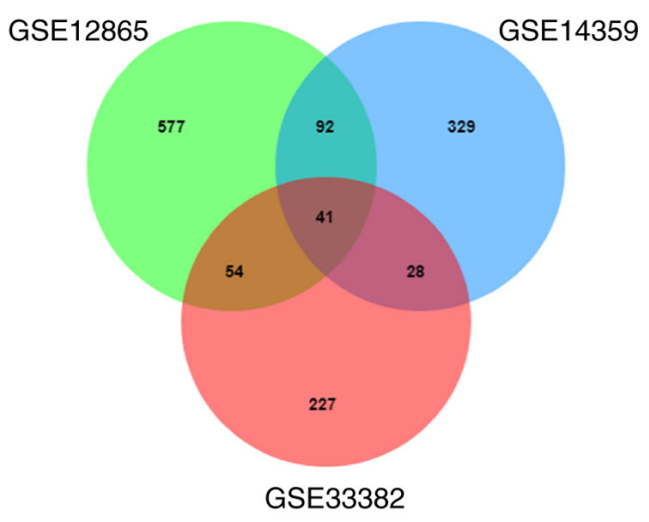

B

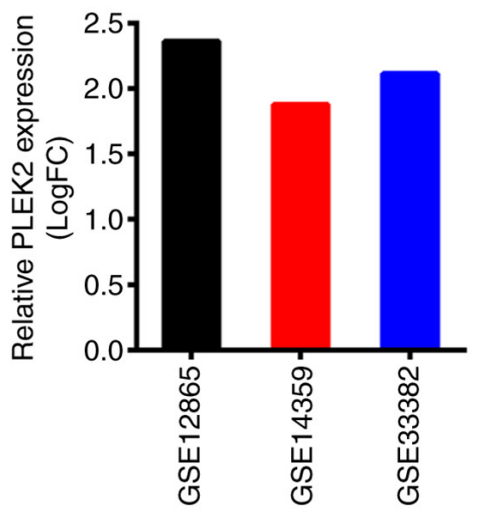

C

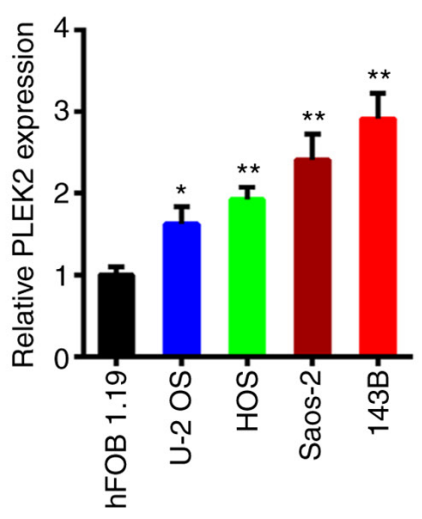

$\mathrm{E}$

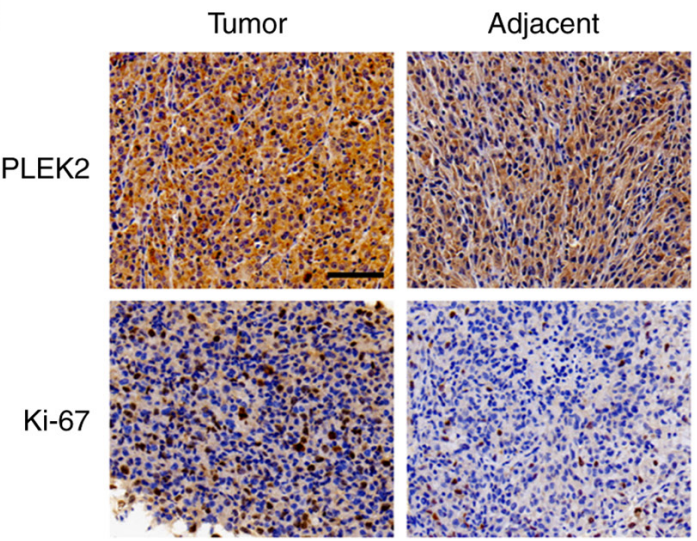

Figure 1. PLEK2 expression is upregulated in OS. (A) The overlapping differentially expressed genes were identified in the three Gene Expression Omnibus datasets. (B) The fold change in PLEK2 expression in OS tissues compared with adjacent normal tissues was analyzed in the three databases. (C) PLEK2 mRNA expression in the four OS cell lines and hFOB 1.19 cells. (D) PLEK2 protein expression in the four OS cell lines, hFOB 1.19 cells and OS tissues. (E) Immunohistochemistry analysis was performed to detect PLEK2 protein expression in OS (scale bar, $100 \mu \mathrm{m}$ ). ${ }^{*} \mathrm{P}<0.05$, ${ }^{* *} \mathrm{P}<0.01 \mathrm{vs}$. hFOB1.19. PLEK2, pleckstrin-2; OS, osteosarcoma; T, tumor tissue; A, adjacent normal tissue.

PLEK2 promotes OS proliferation in vitro and in vivo. Given that PLEK2 expression is different in the four OS cell lines assessed in the present study, and 143B cells exhibited the maximum level, whereas U-2 OS exhibited the minimum level, these cell lines were selected to comprehensively investigate the effects of PLEK2 on OS proliferation, based on previous studies (18-22). U-2 OS and 143B cells were transfected with a PLEK2-coding sequence and shRNA targeting PLEK2, respectively, and the mRNA and protein levels of PLEK2 following transfection were detected via RT-qPCR for knockdown $(\mathrm{P}=0.0002)$ and overexpression $(\mathrm{P}=0.0046$; Fig. 2A) and western blot analyses (Fig. 2B). The CCK-8, colony formation and EdU assays were subsequently performed. As presented in Fig. 2C, PLEK2 knockdown significantly inhibited OS cell proliferation $(\mathrm{P}<0.0001)$, whereas overexpression of PLEK2 promoted OS cell proliferation $(\mathrm{P}<0.0001)$. In addition, PLEK2 knockdown suppressed the colony formation of OS cells $(\mathrm{P}=0.0038)$, whereas overexpression of PLEK2 increased the number of cell colonies compared with the control group $(\mathrm{P}=0.0018$; Fig. 2D). The results of the EdU assay demonstrated that PLEK2 knockdown decreased the percentage of mitotic cells $(\mathrm{P}=0.0018)$, the effects of which were reversed following overexpression of PLEK2 ( $\mathrm{P}=0.0153$; Fig. 2E).
A subcutaneous tumor model was established to better understand the role of PLEK2 in OS in vivo. The results demonstrated that PLEK2 knockdown significantly decreased tumor volume and suppressed OS growth $(\mathrm{P}<0.0001$; Fig. 2F and G). Furthermore, the tumor nodes retrieved from the PLEK2-knockdown group were much smaller compared with the control group, with a lower average tumor weight (Fig. 2H and I). Western blotting verified that PLEK2 expression was suppressed in the sh-PLEK2 group (Fig. 2J). Immunohistochemistry analysis demonstrated that PLEK2 knockdown markedly downregulated Ki-67 expression (Fig. 2K).

PLEK2 promotes OS metastasis in vitro. The Transwell assay was performed to investigate the role of PLEK2 during OS metastasis. The results demonstrated that PLEK2 knockdown suppressed the migratory and invasive abilities of $143 \mathrm{~B}$ ( $\mathrm{P}=0.0005$ for migration and $\mathrm{P}=0.0018$ for invasion), whereas overexpression of PLEK2 promoted cell migration and invasion $(\mathrm{P}=0.001$ for migration and $\mathrm{P}=0.0016$ for invasion, Fig. 3A and B). In addition, the wound healing assay indicated that PLEK2 knockdown decreased the migration distance of 143B cells $(\mathrm{P}=0.0082)$, whereas overexpression of PLEK2 enhanced motility of U-2 OS cells ( $\mathrm{P}=0.0007$; Fig. 3C). 
A
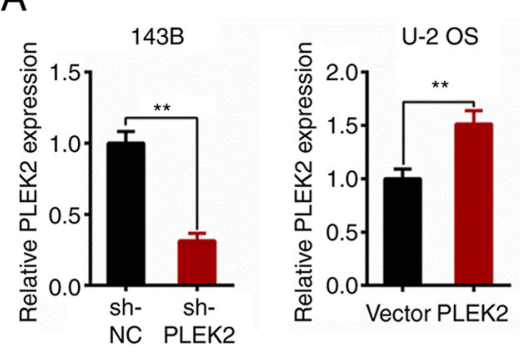

D

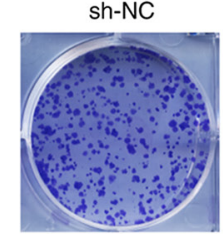

Vector

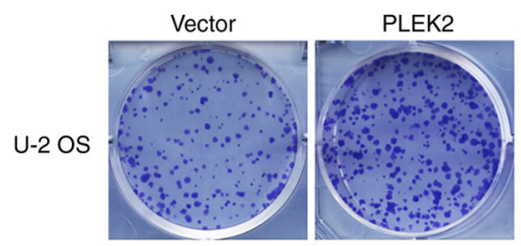

$\mathrm{F}$

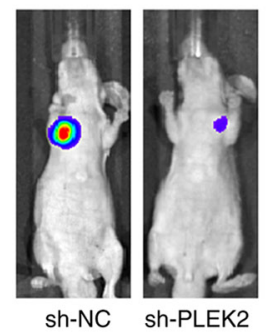

sh-PLEK2

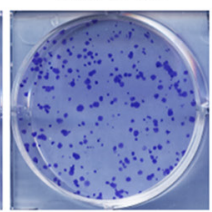

G
B

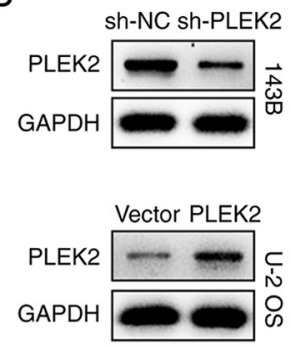

C

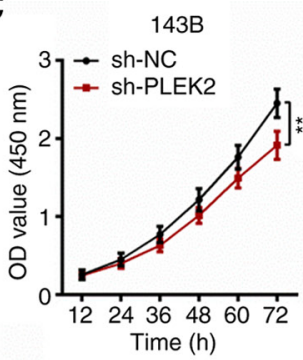

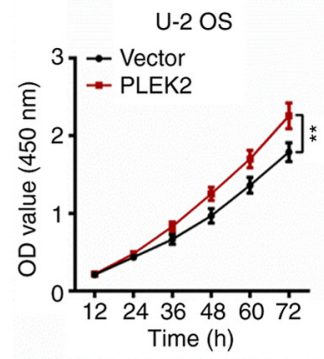

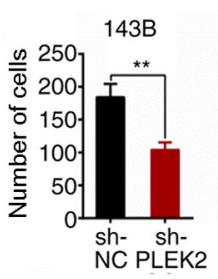

$\mathrm{U}-2$ OS

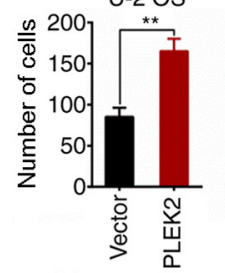

$\mathrm{H}$

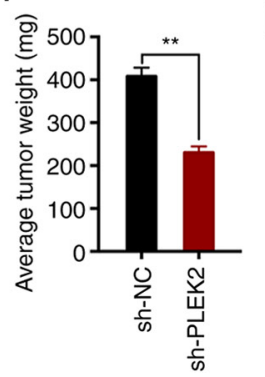

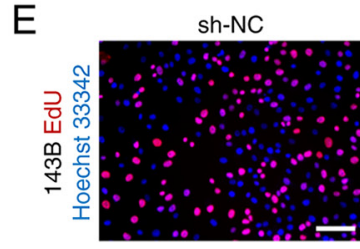

Vector
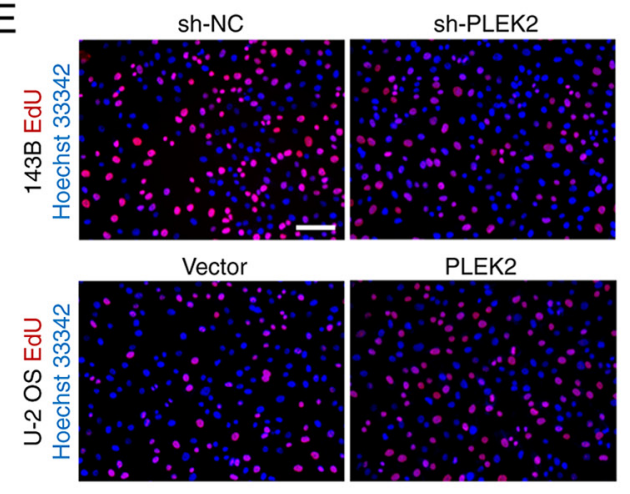

PLEK2
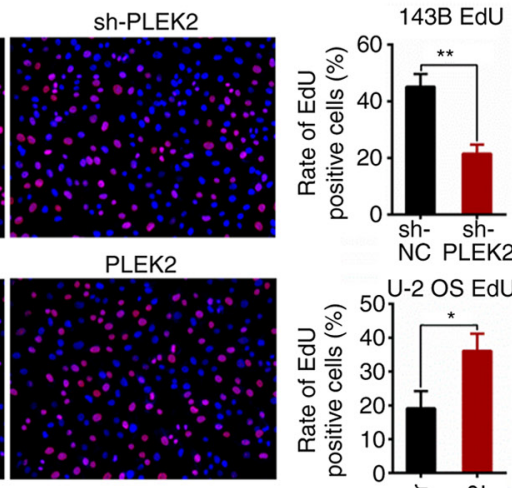

NC PLEK2

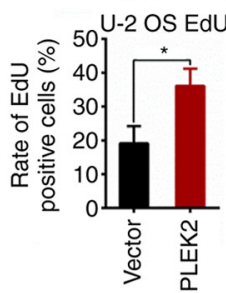

$\mathrm{K}$
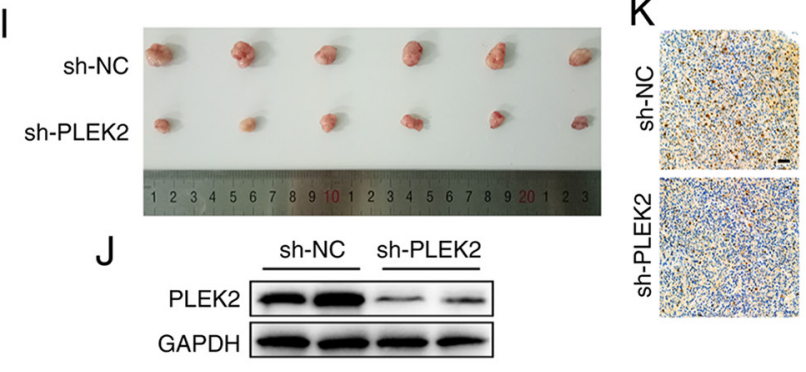

Figure 2. PLEK2 promotes OS proliferation in vitro and in vivo. The transfection efficiency of PLEK2 knockdown and overexpression was determined via (A) reverse transcription-quantitative PCR and (B) western blot analyses in 143B and U-2 OS cells. (C) The effect of PLEK2 on OS cell proliferation was assessed via the Cell Counting Kit- 8 assay at the indicated time points. (D) The cell colony formation assay was performed following PLEK2 overexpression or depletion. (E) The role of PLEK2 in cell proliferation was assessed via the EdU assay (scale bar, $100 \mu \mathrm{m}$ ). (F) Representative images of tumor nodules in the sh-NC and sh-PLEK2 groups. (G) The tumor volumes in the sh-NC and sh-PLEK2 groups were measured at the indicated times following injection until 28 days. (H) Average tumor weights were measured after the mice were sacrificed. (I) Representative images of tumor nodules were taken after the mice were sacrificed. (J) Western blot analysis was performed to detect PLEK2 protein expression in tumor tissues. (K) Ki-67 expression was detected in tumor nodules via immunohistochemistry analysis (scale bar, $100 \mu \mathrm{m}$ ). ${ }^{*} \mathrm{P}<0.05,{ }^{* *} \mathrm{P}<0.01$. PLEK2, pleckstrin-2; OS, osteosarcoma; sh, short hairpin, NC, negative control; OD, optical density.

The EMT process plays a crucial role in tumor metastasis (23); thus, the present study analyzed the expression levels of several EMT-related proteins in OS cells following PLEK2 overexpression or depletion. The results demonstrated that PLEK2 knockdown attenuated the expression levels of MMP-2, N-cadherin and vimentin, the effects of which were reversed following overexpression of PLEK2 (Fig. 3D), which suggests that PLEK2 activates the EMT process.

PLEK2 promotes the EMT process by activating the PI3K/ $A K T / m T O R$ pathway. To investigate the underlying molecular mechanism by which PLEK2 induces the EMT process, single-gene GSEA was performed, which indicated that the PI3K/AKT/mTOR signaling pathway was activated following overexpression of PLEK2 (Fig. 4A and B). Western blot analysis confirmed that PLEK2 knockdown significantly suppressed activation of the PI3K/AKT/mTOR pathway by decreasing the phosphorylation of PI3K and AKT, which resulted in the suppression of EMT. However, the addition of insulin-like growth factor 1, an agonist of the PI3K/ AKT pathway (24), was able to reverse the effects of PLEK2 knockdown. In addition, overexpression of PLEK2 activated the PI3K/AKT/mTOR pathway and EMT process, which was reversed by LY 294002, an inhibitor of the PI3K/AKT pathway (25) (Fig. 4C).

\section{Discussion}

OS is derived from primitive mesenchymal cells, and mainly originates in bones, with soft tissue origination occurring only rarely (26). OS is a high-grade tumor that represents a primary bone malignancy and can be fatal in children, adolescents and young adults (27). PLEK2 has been demonstrated to be upregulated in gallbladder carcinoma 
A

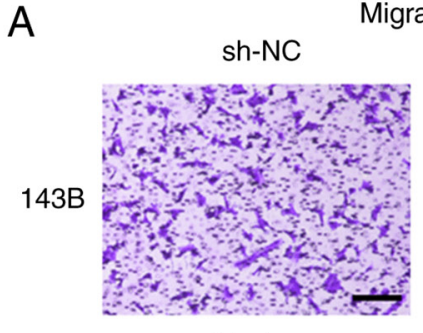

Vector

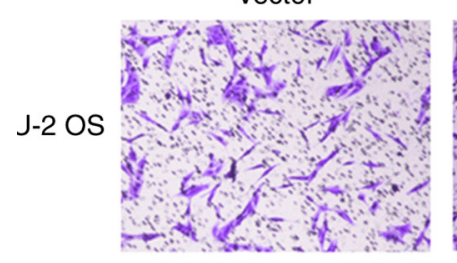

B

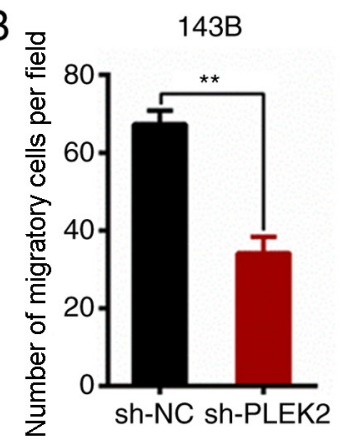

Sh-PLEK2

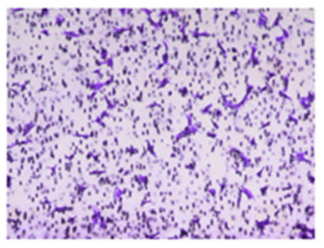

PLEK2

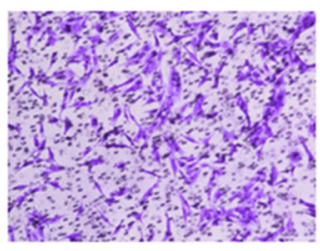

143B

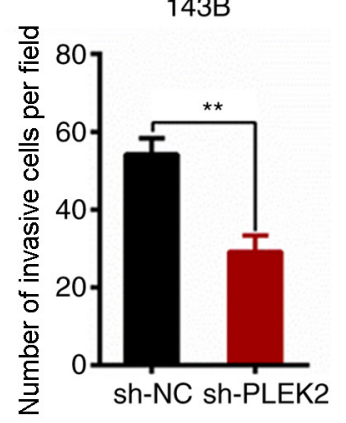

Invasion

sh-NC

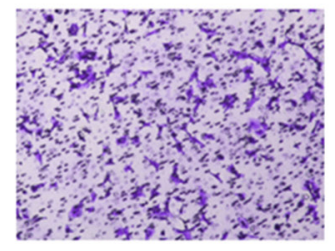

Vector

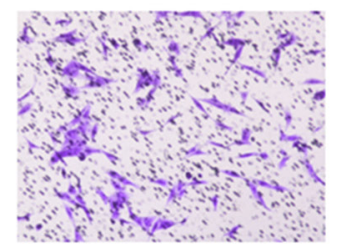

$\mathrm{U}-2$ OS

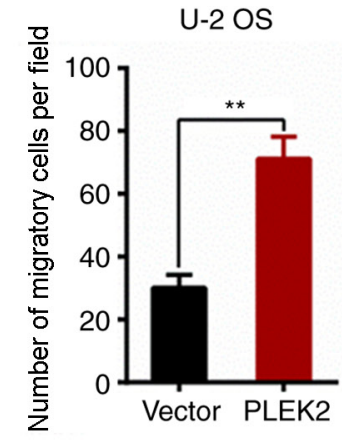

sh-PLEK2

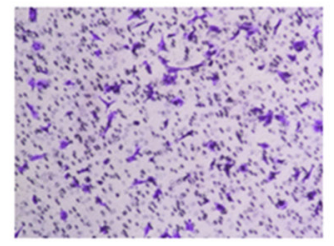

PLEK2

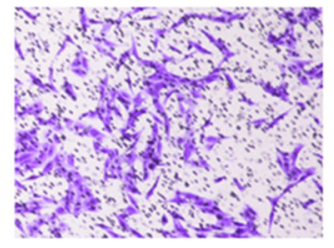

U-2 OS

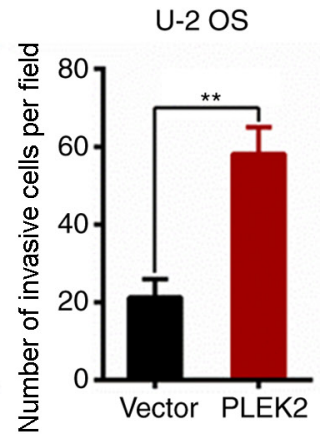

C
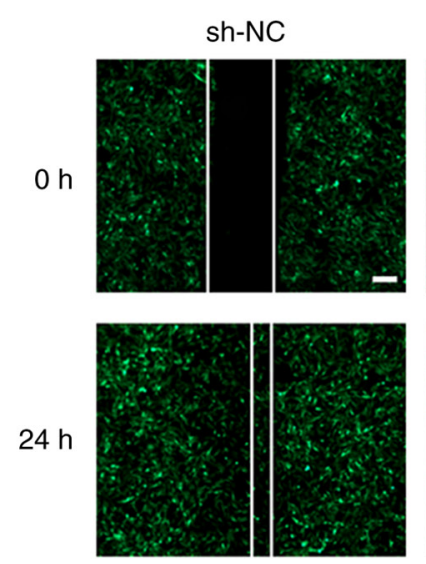

sh-PLEK2
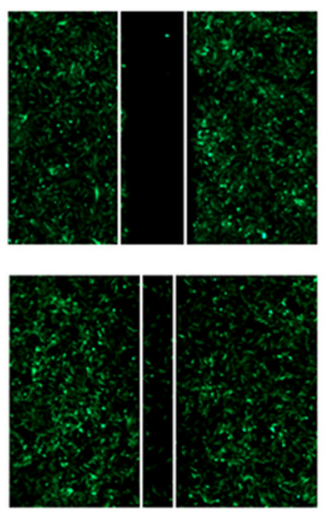
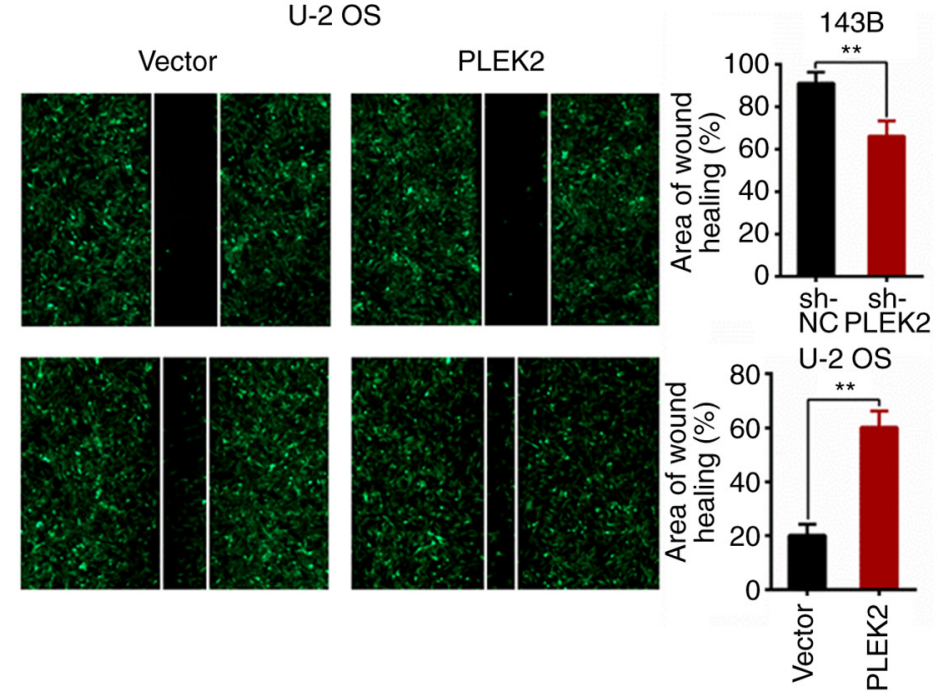

$\mathrm{U}-2$ OS

D

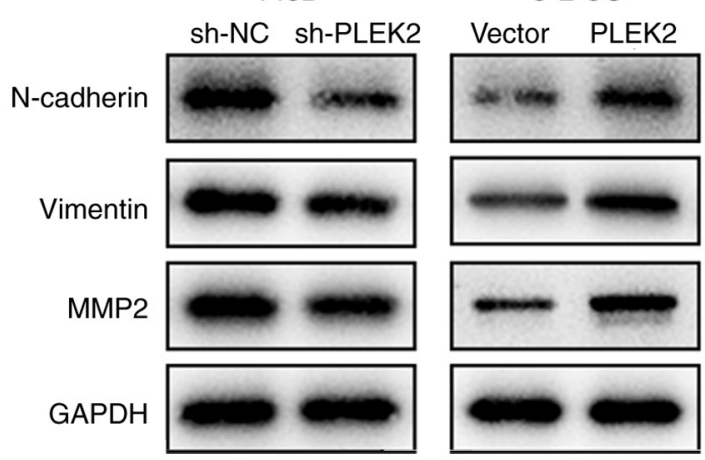

Figure 3. PLEK2 promotes OS metastasis in vitro by activating the EMT process. (A and B) The Transwell assay was performed to assess the effect of PLEK2 on OS cell migration (left) and invasion (right). Scale bar, $100 \mu \mathrm{m}$. (C) The migration distance of OS cells following PLEK2 overexpression or knockdown was measured at the indicated time points (scale bar, $100 \mu \mathrm{m}$ ). (D) Changes in the levels of proteins associated with the EMT process. " P $<0.01$. PLEK2, pleckstrin-2; OS, osteosarcoma; EMT, epithelial-to-mesenchymal transition; sh, short hairpin; NC, negative control; MMP, matrix metalloproteinase. 
A

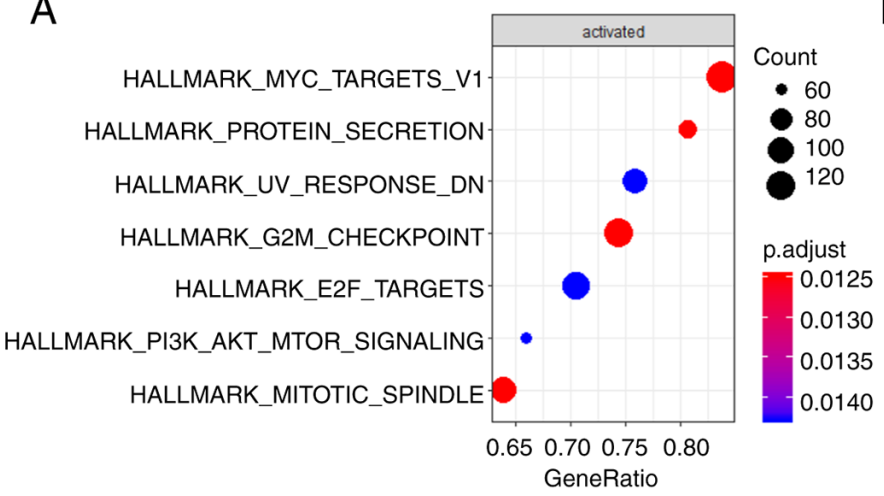

C

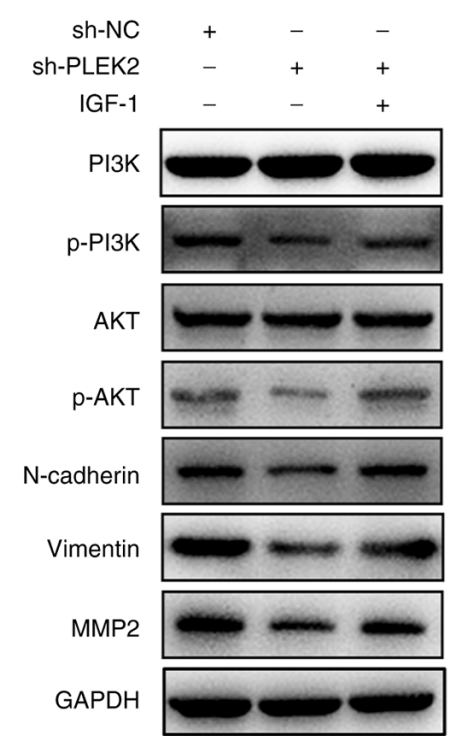

B
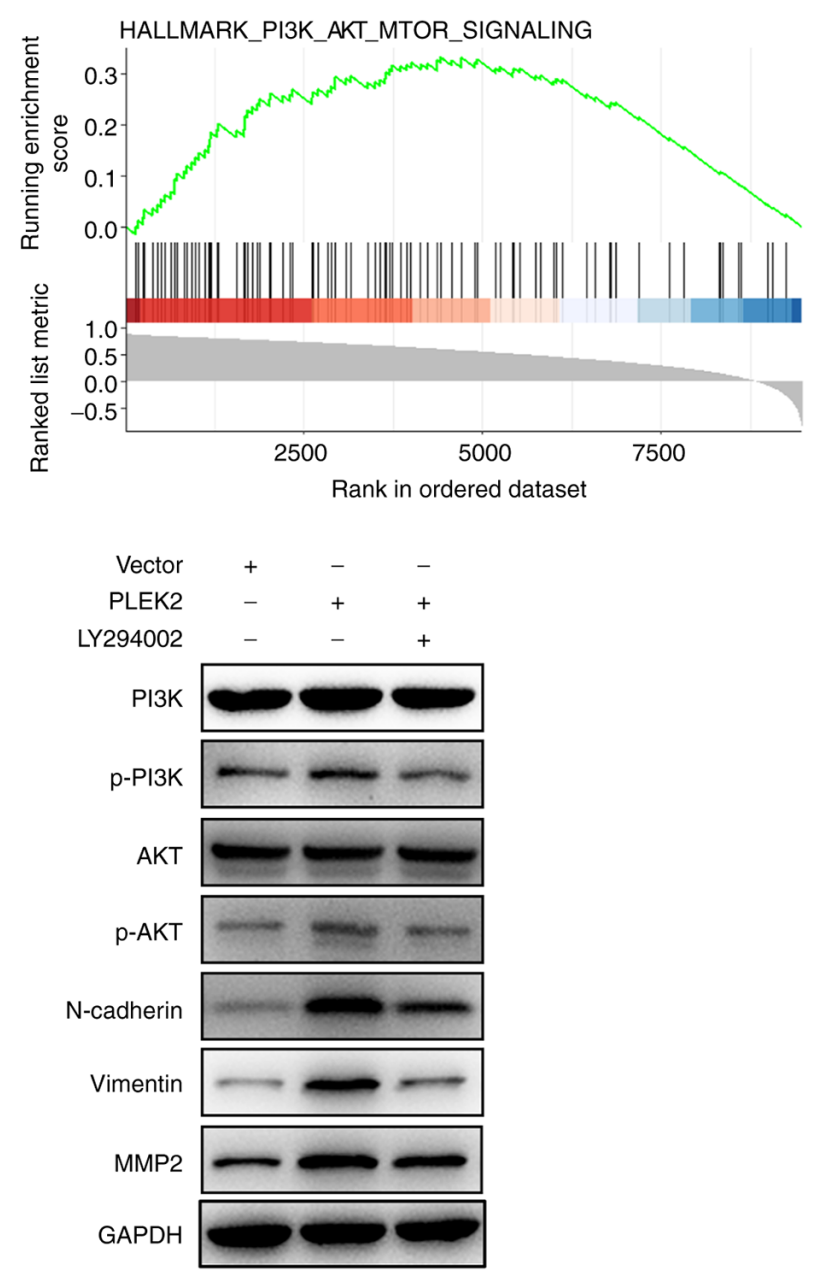

Figure 4. PLEK2 is involved in the PI3K/AKT/mTOR signaling pathway. (A) The top seven enriched signaling pathways associated with overexpression of PLEK2 were identified by single-gene gene set enrichment analysis. (B) The enrichment graph of the PI3K/AKT/mTOR signaling pathway following overexpression of PLEK2. (C) Changes in the expression levels of proteins associated with the PI3K/AKT/mTOR signaling pathway and the epithelial-to-mesenchymal transition process, with the addition of an agonist or inhibitor of the PI3K/AKT/mTOR pathway. PLEK2, plekstrin-2; PI3K, phosphoinositide 3-kinase; AKT, protein kinase B; mTOR, mechanistic target of rapamycin; sh, short hairpin; NC, negative control; IGF-1, insulin-like growth factor 1 ; p, phosphorylated.

tissues, which is associated with the TNM stage and liver metastasis (12). In addition, PLEK2 expressionis positively associated with breast cancer dissemination to the bone marrow (10). PLEK2 induces cellular spreading, causing the formation of large lamellipodia with ruffles by regulating cytoskeletal rearrangement $(28,29)$. The movements of lamellipodia are principal mediators of the EMT process; thus, PLEK2 is considered a dominant driver of cellular metastasis $(30,31)$. Based on previous studies and bioinformatics analyses $(9,11,12)$, the present study investigated the role of PLEK2 in OS and demonstrated that PLEK2 expression was upregulated in OS tissues and cell lines.

The EMT process is closely associated with cancer progression and metastasis. The molecular changes that occur in epithelial cancer cells are characteristic of EMT, including the acquisition of mesenchymal qualities and the loss of epithelial features $(7,23)$. These primary features enhance cancer cell migration and invasion, and increase antitumoral therapy resistance (32). In addition, upregulation of $\mathrm{N}$-cadherin and vimentin, and downregulation of E-cadherin are significant EMT signals (33). In the present study, E-cadherin expression decreased, while vimentin and N-cadherin expression levels increased following overexpression of PLEK2, which promoted tumor progression and metastasis. According to the in vitro Transwell and wound healing assays, the OS cell metastasis capacity was also promoted following overexpression of PLEK2, suggesting that PLEK2 is a likely risk factor for OS progression.

Environmental signals regulate movement and shape cells through large protein assemblies at the plasma membrane that interact with the actin cytoskeleton (34). This regulatory process involves proteins downstream from PI3K that directly control actin polymerization $(35,36)$. These sets of proteins regulate cell protrusions and are important for changing the cellular shape and facilitating cellular spread (37). The PI3K/ AKT/mTOR pathway is an important regulator of cell survival during cellular stress conditions (38). Tumors typically occur in a stressful environment, indicating that the PI3K/AKT/mTOR pathway plays a crucial role in cancer progression. Somatic mutations and gains and losses in key genes are among the abundant genetic alterations that can affect these pathways in a variety of solid and hematological tumors $(39,40)$. Activation 
of the PI3K/AKT/mTOR pathway disrupts cell proliferation and survival regulation, providing tumor cells with a competitive growth advantage and inducing angiogenesis, metastatic competence and therapy resistance (41). Thus, the present study investigated whether PLEK2 activates the PI3K/AKT/ mTOR pathway. The results demonstrated that PLEK2 knockdown decreased the expression levels of phosphorylated PI3K, AKT and EMT-related proteins, the effects of which were reversed following treatment with a PI3K/AKT/ mTOR pathway agonist. Overexpression of PLEK2 reversed these effects in OS cells, suggesting that PLEK2 promotes OS metastasis by inducing the EMT process, mediated by activation of the PI3K/AKT pathway. A limitation of the present study is the lack of follow-up data, which may be helpful to verify the role of PLEK2 in OS.

In conclusion, the results of the present study demonstrated that PLEK2 expression was upregulated in OS, and played a crucial role in OS progression. Furthermore, PLEK2 promoted the EMT process by activating the PI3K/ AKT/mTOR signaling pathway. Taken together, these results suggest that PLEK2 mediates the promotion of tumorigenesis and metastasis, both in vitro and in vivo. PLEK2 promotes the EMT process by targeting the PI3K/AKT/mTOR signaling pathway, promoting metastasis and OS progression. Thus, these results suggest that the inhibition of PLEK2 may represent a potential therapeutic target for OS.

\section{Acknowledgements}

Not applicable.

\section{Funding}

The present study was supported by the Six Talent Peaks Project in Jiangsu Province (grant no. WSW-099).

\section{Availability of data and materials}

All data generated or analyzed during this study are included in this published article.

\section{Authors' contributions}

YL, SY, FW, ZZ, WX, JX, LQ and YG contributed to data analysis, drafting and revised the manuscript for important intellecutual content. YL and YG confirmed the authenticity of all the raw data. All authors have read and approved the final version of the manuscript, and agree to be accountable for all aspects of the work.

\section{Ethics approval and consent to participate}

The present study was approved by the Ethics Committee of the Affiliated Wuxi No. 2 People's Hospital of Nanjing Medical University (Wuxi, China; approval no. 2019-01-0202-05) and written informed consent was provided by patients or their legal guardians prior to the study start. All in vivo animal experiments were approved by the Institutional Animal Care and Use Committee of the Affiliated Wuxi No. 2 People's Hospital of Nanjing Medical University.

\section{Patient consent for publication}

Not applicable.

\section{Competing interests}

The authors declare that they have no competing interests.

\section{References}

1. Wittig JC, Bickels J, Priebat D, Jelinek J, Kellar-Graney K, Shmookler B and Malawer MM: Osteosarcoma: A multidisciplinary approach to diagnosis and treatment. Am Fam Physician 65: 1123-1132, 2002.

2. Bielack SS, Kempf-Bielack B, Delling G, Exner GU, Flege S, Helmke K, Kotz R, Salzer-Kuntschik M, Werner M, Winkelmann W, et al: Prognostic factors in high-grade osteosarcoma of the extremities or trunk: An analysis of 1,702 patients treated on neoadjuvant cooperative osteosarcoma study group protocols. J Clin Oncol 20: 776-790, 2002.

3. Isakoff MS, Bielack SS, Meltzer P and Gorlick R: Osteosarcoma: Current treatment and a collaborative pathway to success. J Clin Oncol 33: 3029-3035, 2015.

4. Link MP, Goorin AM, Miser AW, Green AA, Pratt CB, Belasco JB, Pritchard J, Malpas JS, Baker AR, Kirkpatrick JA, et al: The effect of adjuvant chemotherapy on relapse-free survival in patients with osteosarcoma of the extremity. N Engl J Med 314: 1600-1606, 1986.

5. Meyers PA, Healey JH, Chou AJ, Wexler LH, Merola PR, Morris CD, Laquaglia MP, Kellick MG, Abramson SJ and Gorlick R: Addition of pamidronate to chemotherapy for the treatment of osteosarcoma. Cancer 117: 1736-1744, 2011.

6. Hanahan D and Coussens LM: Accessories to the crime: Functions of cells recruited to the tumor microenvironment. Cancer Cell 21: 309-322, 2012.

7. Taki M, Abiko K, Ukita M, Murakami R, Yamanoi K, Yamaguchi K, Hamanishi J, Baba T, Matsumura N and Mandai M: Tumor immune microenvironment during epithelial-mesenchymal transition. Clin Cancer Res: Apr 7, 2021 (Epub ahead of print).

8. Lamouille S, Xu J and Derynck R: Molecular mechanisms of epithelial-mesenchymal transition. Nat Rev Mol Cell Biol 15: 178-196, 2014.

9. Hu MH, Bauman EM, Roll RL, Yeilding $\mathrm{N}$ and Abrams CS: Pleckstrin 2, a widely expressed paralog of pleckstrin involved in actin rearrangement. J Biol Chem 274: 21515-21518, 1999.

10. Naume B, Zhao X, Synnestvedt M, Borgen E, Russnes HG, Lingjaerde OC, Strømberg M, Wiedswang G, Kvalheim G, Kåresen R, et al: Presence of bone marrow micrometastasis is associated with different recurrence risk within molecular subtypes of breast cancer. Mol Oncol 1: 160-171, 2007.

11. Luo Y, Robinson S, Fujita J, Siconolfi L, Magidson J, Edwards CK, Wassmann K, Storm K, Norris DA, Bankaitis-Davis D, et al: Transcriptome profiling of whole blood cells identifies PLEK2 and C1QB in human melanoma. PLoS One 6: e20971, 2011.

12. Shen H, He M, Lin R, Zhan M, Xu S, Huang X, Xu C, Chen W, Yao Y, Mohan M and Wang J: PLEK2 promotes gallbladder cancer invasion and metastasis through EGFR/CCL2 pathway. J Exp Clin Cancer Res 38: 247, 2019.

13. Sadikovic B, Yoshimoto M, Chilton-MacNeill S, Thorner P, Squire JA and Zielenska M: Identification of interactive networks of gene expression associated with osteosarcoma oncogenesis by integrated molecular profiling. Hum Mol Genet 18: 1962-1975, 2009.

14. Fritsche-Guenther R, Noske A, Ungethüm U, Kuban RJ, Schlag PM, Tunn PU, Karle J, Krenn V, Dietel M and Sers C: De novo expression of EphA2 in osteosarcoma modulates activation of the mitogenic signalling pathway. Histopathology 57: 836-850, 2010.

15. Kuijjer ML, Rydbeck H, Kresse SH, Buddingh EP, Lid AB, Roelofs $\mathrm{H}$, Bürger $\mathrm{H}$, Myklebost $\mathrm{O}$, Hogendoorn PC, Meza-Zepeda LA and Cleton-Jansen AM: Identification of osteosarcoma driver genes by integrative analysis of copy number and gene expression data. Genes Chromosomes Cancer 51: 696-706, 2012 . 
16. Livak KJ and Schmittgen TD: Analysis of relative gene expression data using real-time quantitative PCR and the 2(-Delta Delta C(T)) method. Methods 25: 402-408, 2001.

17. Laferriere CA and Pang DS: Review of intraperitoneal injection of sodium pentobarbital as a method of euthanasia in laboratory rodents. J Am Assoc Lab Anim Sci 59: 254-263, 2020.

18. Ge X, Liu W, Zhao W, Feng S, Duan A, Ji C, Shen K, Liu W, Zhou J, Jiang D, et al: Exosomal transfer of LCP1 promotes osteosarcoma cell tumorigenesis and metastasis by activating the JAK2/STAT3 signaling pathway. Mol Ther Nucleic Acids 21: 900-915, 2020

19. Kong Y, Wu R, Zhang S, Zhao M, Wu H, Lu Q, Fu S and Su Y: Wilms' tumor 1-associating protein contributes to psoriasis by promoting keratinocytes proliferation via regulating cyclin A2 and CDK2. Int Immunopharmacol 88: 106918, 2020.

20. Lin L, Xiao J, Shi L, Chen W, Ge Y, Jiang M, Li Z, Fan H, Yang L and Xu Z: STRA6 exerts oncogenic role in gastric tumorigenesis by acting as a crucial target of miR-873. J Exp Clin Cancer Res 38: 452, 2019.

21. Liu W, Jiang D, Gong F, Huang Y, Luo Y, Rong Y, Wang J, Ge X, Ji C, Fan J and Cai W: miR-210-5p promotes epithelial-mesenchymal transition by inhibiting PIK3R5 thereby activating oncogenic autophagy in osteosarcoma cells. Cell Death Dis 11: 93, 2020.

22. Shen T, Wang W, Zhou W, Coleman I, Cai Q, Dong B, Ittmann MM, Creighton CJ, Bian Y, Meng Y, et al: MAPK4 promotes prostate cancer by concerted activation of androgen receptor and AKT. J Clin Invest 131: e135465, 2021.

23. Bakir B, Chiarella AM, Pitarresi JR and Rustgi AK: EMT, MET, plasticity, and tumor metastasis. Trends Cell Biol 30: 764-776, 2020

24. Cheng HC, Chang TK, Su WC, Tsai HL and Wang JY: Narrative review of the influence of diabetes mellitus and hyperglycemia on colorectal cancer risk and oncological outcomes. Transl Oncol 14: 101089, 2021

25. Jiang J, Xu Y, Ren H, Wudu M, Wang Q, Song X, Su H, Jiang X, Jiang $L$ and Qiu X: MKRN2 inhibits migration and invasion of non-small-cell lung cancer by negatively regulating the PI3K/ Akt pathway. J Exp Clin Cancer Res 37: 189, 2018.

26. Kim EH, Kim MS, Lee KH, Sai S, Jeong YK, Koh JS and Kong CB: Effect of low- and high-linear energy transfer radiation on in vitro and orthotopic in vivo models of osteosarcoma by activation of caspase-3 and -9. Int J Oncol 51: 1124-1134, 2017.

27. Biermann JS, Adkins DR, Agulnik M, Benjamin RS, Brigman B, Butrynski JE, Cheong D, Chow W, Curry WT, Frassica DA, et al: Bone cancer. J Natl Compr Canc Netw 11: 688-723, 2013.

28. Bach TL, Kerr WT, Wang Y, Bauman EM, Kine P, Whiteman EL, Morgan RS, Williamson EK, Ostap EM, Burkhardt JK, et al: $\mathrm{PI} 3 \mathrm{~K}$ regulates pleckstrin-2 in T-cell cytoskeletal reorganization. Blood 109: 1147-1155, 2007.
29. Hamaguchi N, Ihara S, Ohdaira T, Nagano H, Iwamatsu A, Tachikawa $\mathrm{H}$ and Fukui Y: Pleckstrin-2 selectively interacts with phosphatidylinositol 3-kinase lipid products and regulates actin organization and cell spreading. Biochem Biophys Res Commun 361: 270-275, 2007.

30. Yilmaz $\mathrm{M}$ and Christofori G: EMT, the cytoskeleton, and cancer cell invasion. Cancer Metastasis Rev 28: 15-33, 2009.

31. Ombrato L and Malanchi I: The EMT universe: Space between cancer cell dissemination and metastasis initiation. Crit Rev Oncog 19: 349-361, 2014.

32. Smith BN and Bhowmick NA: Role of EMT in metastasis and therapy resistance. J Clin Med 5: 17, 2016.

33. Wu Y, Sarkissyan M and Vadgama JV: Epithelial-mesenchymal transition and breast cancer. J Clin Med 5: 13, 2016.

34. Chaki SP, Barhoumi R, Berginski ME, Sreenivasappa H, Trache A, Gomez SM and Rivera GM: Nck enables directional cell migration through the coordination of polarized membrane protrusion with adhesion dynamics. J Cell Sci 126: 1637-1649, 2013.

35. Lafuente EM, van Puijenbroek AA, Krause M, Carman CV, Freeman GJ, Berezovskaya A, Constantine E, Springer TA, Gertler FB and Boussiotis VA: RIAM, an Ena/VASP and profilin ligand, interacts with Rap1-GTP and mediates Rap1-induced adhesion. Dev Cell 7: 585-595, 2004

36. Krause M, Leslie JD, Stewart M, Lafuente EM, Valderrama F, Jagannathan R, Strasser GA, Rubinson DA, Liu H, Way M, et al: Lamellipodin, an Ena/VASP ligand, is implicated in the regulation of lamellipodial dynamics. Dev Cell 7: 571-583, 2004.

37. Tscharntke M, Pofahl R, Krieg T and Haase I: Ras-induced spreading and wound closure in human epidermal keratinocytes. FASEB J 19: 1836-1838, 2005.

38. Datta SR, Brunet A and Greenberg ME: Cellular survival: A play in three Akts. Genes Dev 13: 2905-2927, 1999.

39. Ediriweera MK, Tennekoon KH and Samarakoon SR: Role of the PI3K/AKT/mTOR signaling pathway in ovarian cancer: Biological and therapeutic significance. Semin Cancer Biol 59: 147-160, 2019.

40. Hoxhaj G and Manning BD: The PI3K-AKT network at the interface of oncogenic signalling and cancer metabolism. Nat Rev Cancer 20: 74-88, 2020.

41. Porta C, Paglino C and Mosca A: Targeting PI3K/Akt/mTOR signaling in cancer. Front Oncol 4: 64, 2014.

(i) $($ ) This work is licensed under a Creative Commons (c) NC No Attribution-NonCommercial-NoDerivatives 4.0 International (CC BY-NC-ND 4.0) License. 